\title{
Perspectiva campesina, intoxicaciones por plaguicidas y uso de agroquímicos
}

\author{
Peasant perspective, poisoning by pesticide and agrochemical usage \\ Paulina Guzmán-Plazola1, Rubén Darío Guevara-Gutiérrez" ${ }^{*}$, \\ José Luis Olguín-López, Oscar Raúl Mancilla-Villa ${ }^{1}$
}

\begin{abstract}
RESUMEN
Los agroquímicos son sustancias de uso común en las actividades agrícolas para favorecer y mejorar el desarrollo de cultivos e incrementar la producción. Según la Organización de las Naciones Unidas para la Agricultura y la Alimentación (FAO, 1997) 99\% de las intoxicaciones agudas en las actividades agrícolas han sido provocadas por el uso de estas sustancias, ya sea por contacto directo o indirecto, problema que se incrementa debido a la falta de equipo adecuado para su manejo y aplicación. La presente investigación presenta las estadísticas (2001-2010) brindada por el Sector Salud, complementada mediante entrevistas a los agricultores como usuarios de agroquímicos. Durante este periodo a nivel nacional las intoxicaciones agudas por plaguicidas registradas en promedio al año fue de 3.928; a nivel estatal, Jalisco destaca como la principal entidad con mayor incidencia de casos; en éste la región Sierra de Amula entre los años 2004 a 2011 los municipios de Casimiro Castillo y Autlán de Navarro se identifican por el mayor número de casos registrados. En este último municipio de 24 ejidos encuestados, 19 manifiestan el daño a la salud por estos productos; $68 \%$ de los entrevistados que no acuden al centro de atención médica emplean remedios caseros; problemática que es proporcional a las actividades agrícolas y al periodo de lluvia. Los productos con mayor frecuencia empleados son Lannate (Metomilo: S-Metil-N- metilcarbamoil oxi - tioacetamidato), Tamarón (Metamidofos: O, S-Dimetil fosforoamidotioato) y Furadán (Carbofuran: 2, 3-dihidro-2, 2-dimetil-7-benzofuranil-metil carbamato) los que están reconocidos por su mayor grado de toxicidad. El riesgo de sufrir intoxicaciones por agroquímicos está relacionado al desconocimiento técnico, a la falta de capacitación de manejo y uso, así como a la información proporcionada por las casas comerciales; situación que ha generado la conformación de conocimiento empírico entre los agricultores.
\end{abstract}

Palabras clave: agroquímico, plaguicida, Lannate, Tamarón, Furadán.

\begin{abstract}
Agrochemicals are substances commonly used in agricultural activities to promote and enhance the development of crops and increase production. According to FAO (1997) 99\% of acute poisoning in agricultural activities is caused by the use of these substances, either by direct or indirect contact, a problem that increases due to lack of proper equipment for handling and application. This research presents statistics (2001-2010) provided by the Health Sector, complemented by interviews to farmers as users of agrochemicals. Nationally, the number of acute pesticide poisonings registered per year is 3,928; At the State level, Jalisco stands out as the principal entity with higher incidence; inside Jalisco; the "Sierra de Amula" region during the years 2004-2011, the municipalities of Casimiro Castillo and Autlán de Navarro are identified by the largest number of registered cases. In the latter municipality, from 24 surveyed "ejidos", 19 demonstrate the damage to health by these products; 68\% of respondents do not seek medical service, instead they use home remedies; a problem that is proportional to agricultural activities and the rain period. The products most commonly used are Lannate, Tamaron and Furadan which are recognized for their higher degree of toxicity. The risk of poisoning by agrochemicals is related to the lack of technical knowledge, lack of training for management and use, as well as information provided by commercial firms; situation that generated the formation of empirical knowledge among farmers.
\end{abstract}

Key words: agrochemical, pesticide, Lannate, Tamaron, Furadan.

1 Universidad de Guadalajara, Centro Universitario de la Costa Sur, Departamento de Ecología y Recursos Naturales. Autlán de Navarro, Jalisco, México.

* Autor por correspondencia: rguevara@ cucsur.udg.mx

Fecha de Recepción: 31 Agosto, 2015.

Fecha de Aceptación: 13 Mayo, 2016. 


\section{Introducción}

El término plaguicida comprende diferentes productos que tienen como fin específico destruir o controlar plagas y enfermedades, de acuerdo a su función se clasifican en herbicidas, insecticidas, fungicidas, nematicidas y rodenticidas (FAO, 1997). Los agroquímicos son sustancias orgánicas o inorgánicas utilizadas en las actividades agrícolas para favorecer y mejorar los cultivos e incrementar la producción, empleo que se incrementó debido a la demanda mundial por alimentos (granos y cereales principalmente), situación que se ha traducido en el uso indiscriminado de estos productos (Consejo de Salubridad General, 2011).

El uso de estos productos es una práctica común en las labores agrícolas, el uso excesivo los ha convertido en una problemática mundial dada su toxicidad para aquellas personas que los manejan, por encontrarse expuestos continuamente al componente y/o ingrediente activo de dichas sustancias, llegando a causar intoxicaciones que generan signos y síntomas puntuales, hasta dar lugar a secuelas o efectos crónicos (Alvarado y Pérez, 1998; Torres y Capote, 2004). FAO (1997) menciona que 99\% de las intoxicaciones en las actividades agrícolas son provocadas por el uso de agroquímicos. Basado en la Organización de las Naciones Unidas (Montoro et al., 2009) se establece que las causas principales de estas intoxicaciones son la reglamentación, la educación, la comunicación sobre riesgos y la falta de participación en la adopción de decisiones, así como con problemas de disposición de los envases y en el almacenamiento de los agroquímicos. Madeley (2002) menciona que el uso de éstos ha ido en aumento en los últimos 20 años sobre todo en los países en desarrollo en el sector agrícola, en donde se carece de campañas de salud.

El uso de agroquímicos a nivel mundial, ha ocasionado diversos daños o alteraciones en el ambiente y en el ser humano; en éste último, estudios epidemiológicos revelan diversos daños y enfermedades como la hepatitis, mal formaciones congénitas, discapacidad mental, órganos dañados y varios tipos de cáncer como leucemia, cáncer de piel, cáncer de pecho y tumores cerebrales, así como un elevado riesgo de sarcoma de tejidos blandos (Alvarado y Pérez, 1998; Montoro et al., 2009). Adicional a ello Montes et al. (2010) mencionan desórdenes reproductivos en el hombre (disminución de espermatozoides, cáncer testicular y defectos congénitos como criptorquidia e hipospadias), considerando la causa principal la exposición a químicos con actividad estrogénica y antiandrogénica tales como plaguicidas organoclorados, bifenilospoliclorados (PCBs), dioxinas, detergentes y plastificantes, entre otros. Rivero et al. (2001) al respecto menciona que la intensidad de afectación estará ligado a la dosis y tiempo de exposición así como la susceptibilidad de cada organismo.

De acuerdo con la Organización Mundial de la Salud (OMS) los plaguicidas en países en desarrollo, causan un millón de casos de intoxicación y cerca de 20,000 muertes anualmente (Martínez-Valenzuela y Gómez-Arroyo, 2007). Safe (1995), relaciona el carcinoma mamario con algunos productos de exposición ambiental, como son los compuestos organoclorados (insecticidas organoclorados, bifenilospoliclorados y dioxinas). Según INEGI el carcinoma de mama femenina fue el padecimiento responsable de 3.220 defunciones registradas en el año 1997 en México (Waliszewski et al., 2003). Aunque en este sentido las cifras no reflejan la magnitud real del problema, ya que el sub-registro es evidente en las estadísticas así como el uso inadecuado de los registros en las zonas rurales (Montoro et al., 2009). En México, gran parte de la población que está involucrada con el sector agrícola, desde 1993 se reportan casos por intoxicaciones agudas por plaguicidas, lamentablemente estas cifras no muestran la verdadera realidad ya que se calcula que por cada caso registrado, cinco al menos no se contabilizan (Cortés-Genchi et al., 2007). Otros ejemplos son los reportados por Aranguré et al. (2007) y Aguilar et al. (2007), los cuales evaluaron la bioacumulación de pesticidas organoclorados en sedimentos y peces (Cyprinus carpio, Oreochromis aureus, Micropterus salmoides, Ictalurus punctatus y Dorosoma smithi) realizado durante periodos de estiaje y lluvia, en la presa hidroeléctrica Aguamilpa, Estado de Nayarit, México, efecto de la actividad agrícola y pesquera; encontrando que los niveles de estos plaguicidas en filetes de peces fueron menores a los límites máximos permisibles por la Food and Drug Administration (FDA) y el Codex Alimentarius.

En la región Costa Sur y Sierra de Amula en el estado de Jalisco-México, los residuos potencialmente peligrosos que se generan en el territorio son principalmente de origen industrial (residuos líquidos y aerosoles del procesamiento 
de la caña de azúcar y aceites residuales principalmente) o agrícola (pesticidas de amplio espectro y envases de agroquímicos) los cuales se han venido acumulando desde hace 25 años o más por efecto de las actividades agrícolas que se desarrolla dentro de estas regiones, principalmente en los valles de los municipios de El Limón, El Grullo y Autlán de Navarro. Basado en los reportes del sector salud, en este último municipio el reporte de las intoxicaciones por plaguicidas ha venido en aumento, debido a la actividad agrícola, que representa la principal actividad económica ( $19^{\circ}$ lugar en el estado) y registrándose 27 casos de intoxicaciones por plaguicidas al año (Aguilar, 2006).

El desarrollo de la presente investigación fue a través de conocer las perspectivas de la sociedad dedicada a la actividad agrícola de 24 ejidos que conforman el Municipio de Autlán de Navarro en el estado de Jalisco-México, en referencia del uso de productos agroquímicos y el impacto de éstos en la salud humana.

\section{Metodología}

\section{Descripción del área de estudio}

El presente estudio fue desarrollado en el municipio de Autlán de Navarro, ubicado al suroeste del estado de Jalisco (Figura 1), entre los 19³4'30" a $19^{\circ} 53^{\prime} 45^{\prime \prime}$ norte y $104^{\circ} 07^{\prime} 00^{\prime \prime}$ a $104^{\circ} 27^{\prime} 35^{\prime \prime}$ " oeste, sobre una altitud promedio de $900 \mathrm{~m}$, dentro de la cuenca del río Ayuquila - Armería en las estribaciones de la Sierra Volcánica Transversal, por lo que su topografía es irregular, con variación en altitud de 1949 metros (900 a $2.840 \mathrm{msnm}$ ) lo que refleja diversidad climática, presenta clima semiseco con otoño, invierno y primavera secos y semicálidos, sin cambio térmico invernal bien definido, temperatura promedio anual de $23,5^{\circ} \mathrm{C}$ y precipitación anual de 719,8 mm. Los suelos son del tipo Feozem háplico, Vertisol pélico, Andosol, Regosol y Cambisol eútrico. Las actividades económicas son basadas principalmente en la agricultura de riego proyectada sobre la caña de azúcar (Saccharum officinarum),

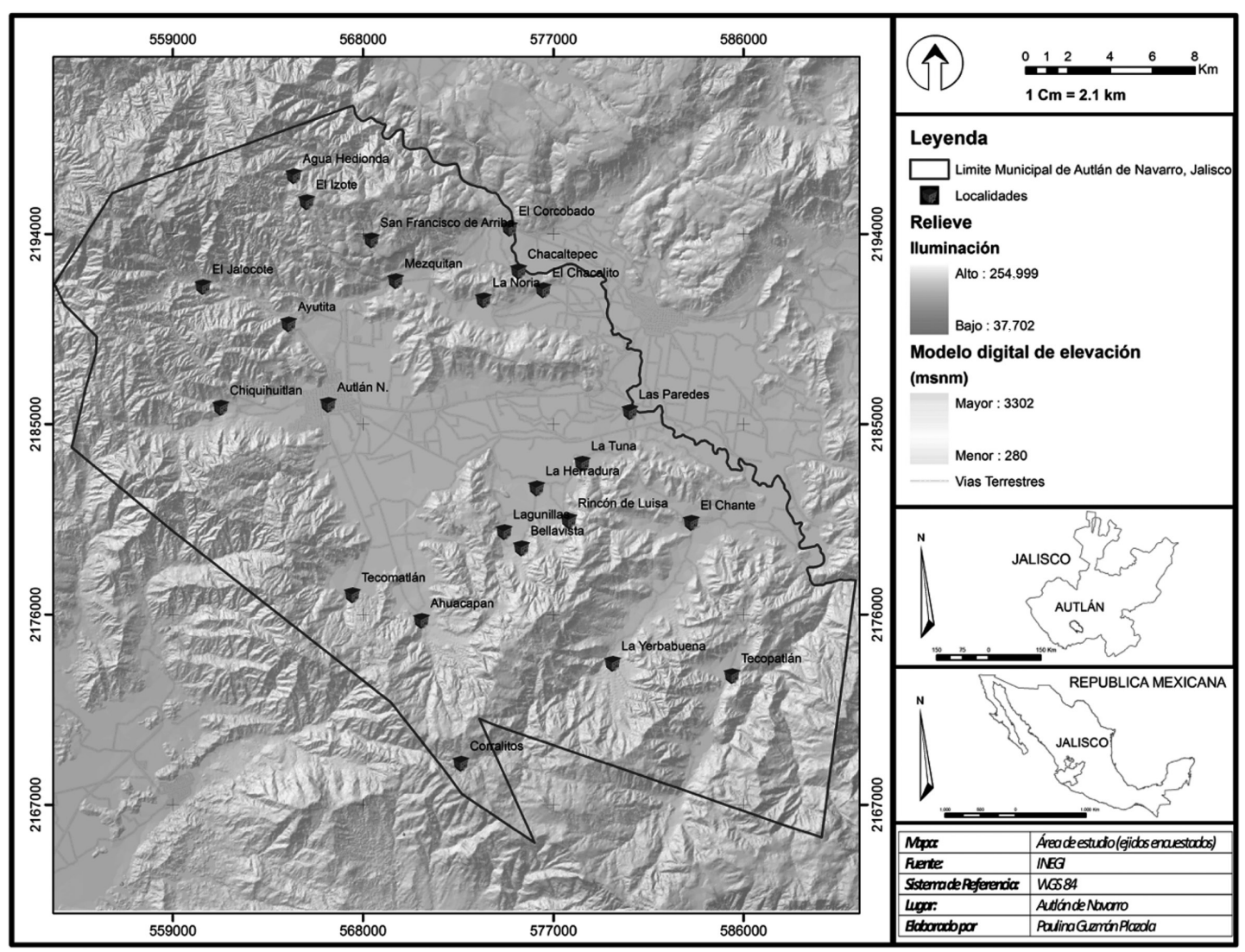

Figura 1. Comunidades consideradas en el área de estudio, Mpio. Autlán de Navarro, Jalisco. 
jitomate (Lycopersicum esculentum) y chile jalapeño (Capsicum annum), así como la agricultura de temporal anual proyectada sobre el maíz (Zea mays) y sorgo (Sorghum bicolor) distribuidos en una superficie de 15.551,7997 has (20,99\% del área total municipal).

\section{Métodos}

El desarrollo de la presente investigación fue a través de conocer las perspectivas de la sociedad dedicada a la actividad agrícola en referencia del uso de productos agroquímicos y el impacto de éstos en la salud humana. Para ello se realizaron entrevistas a los agricultores mediante el método de diagnóstico rápido empleando preguntas abiertas y cerradas, cuestionando aspectos sobre: uso, manejo y aplicación de productos químicos, así como los problemas que éstos les han causado a su salud.

El muestreo fue aleatorio y realizado a 229 agricultores como parte representativa del total para el municipio mediante muestras aleatorias a través de una población finita obtenida mediante la relación:

$$
n=\frac{\frac{N}{e^{2}(N-1)}}{1+z^{2} p q}
$$

Dónde: $n, \quad$ Tamaño de la muestra.

N, Tamaño de la población conocida.

E, $\quad$ Error muestral del $95 \%$ en este caso representado por 1,96 .

$Z, \quad$ Nivel de confianza $\alpha=0,05$. $p q(\sigma 2),(0,05)(0,05)=0,25$.

Adicional a esta información, se reporta la información generada por el sector salud (Instituto Mexicano del Seguro Social y Secretaría de Salubridad y Asistencia Pública) en referencia a los casos registrados de intoxicación aguda por plaguicidas, lográndose obtener la base de datos nacional del número de casos de intoxicaciones de una década (2001 a 2010) y municipal de 6 años (2005 a 2010).

\section{Resultados y Discusión}

\section{Registro de intoxicaciones a nivel nacional}

Basado en los reportes de la Secretaria de Salud Pública (SSP) en específico por el Instituto
Mexicano del Seguro Social (IMSS) de 2001 a 2010 se registraron 31.257 casos de intoxicaciones agudas por plaguicidas que recibieron atención médica a nivel nacional, con promedio anual de 3.126 , valor mínimo de 1.453 y máximo de 3.928 casos al año (Figura 2). De las entrevistas realizadas a los agricultores del municipio de Autlán de Navarro en el estado de Jalisco, el manifiesto sobre la presencia de algún tipo de intoxicación por agroquímicos, $68 \%$ de ellos afirman no acudir a ningún centro de atención médica por considerarlo innecesario, ya que presentan síntomas "leves", siendo solucionados con remedios caseros proporcionados por familiares o por ellos mismos (ingiriendo leche, jugo de limón, bicarbonato o tomando un baño); respuesta que hace suponer que éste es el esquema seguido a nivel nacional y por lo tanto, los registros oficiales del sector salud no representa todos los casos de intoxicación ocurridos.

De esta base de datos, se observa que los casos de intoxicación registrados durante este periodo se incrementaron de junio a septiembre, meses caracterizados por corresponder al periodo de lluvias, siembra de cultivos de temporal y aumento del uso de agroquímicos en las actividades agrícolas. Esto último debido al crecimiento de malezas y con ello, el uso de herbicidas para su control como parte de las labores convencionales realizadas en el municipio. Bajo el supuesto que el ciclo de siembra dominante en gran parte del país es primavera-verano (temporal de lluvias) se infiere que los registros de intoxicaciones a nivel nacional es similar (Figura 3).

De los registros de las entidades federativas durante este periodo, se observó el mayor número de casos por intoxicación a través del tiempo en el estado de Jalisco con una proporción de 4:1 con respecto a otros estados (Chiapas, Guerrero, Michoacán, Nayarit, Sinaloa y Veracruz) que registran mayor incidencia (Figura 4).

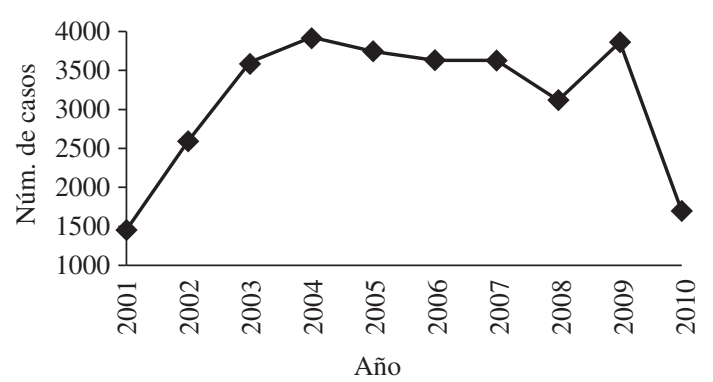

Figura 2. Casos de intoxicaciones agudas por plaguicidas promedio en México (basado de registros del IMSS, 2010). 


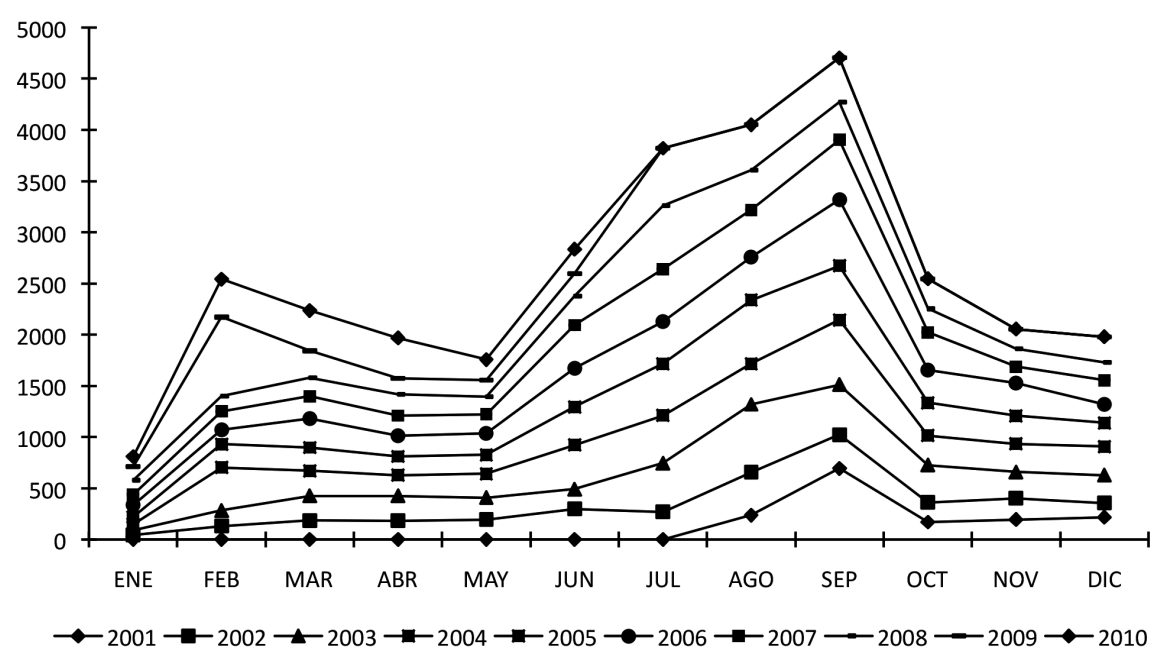

Figura 3. Registro promedio mensual de intoxicaciones a nivel nacional (Sector Salud, 2010).

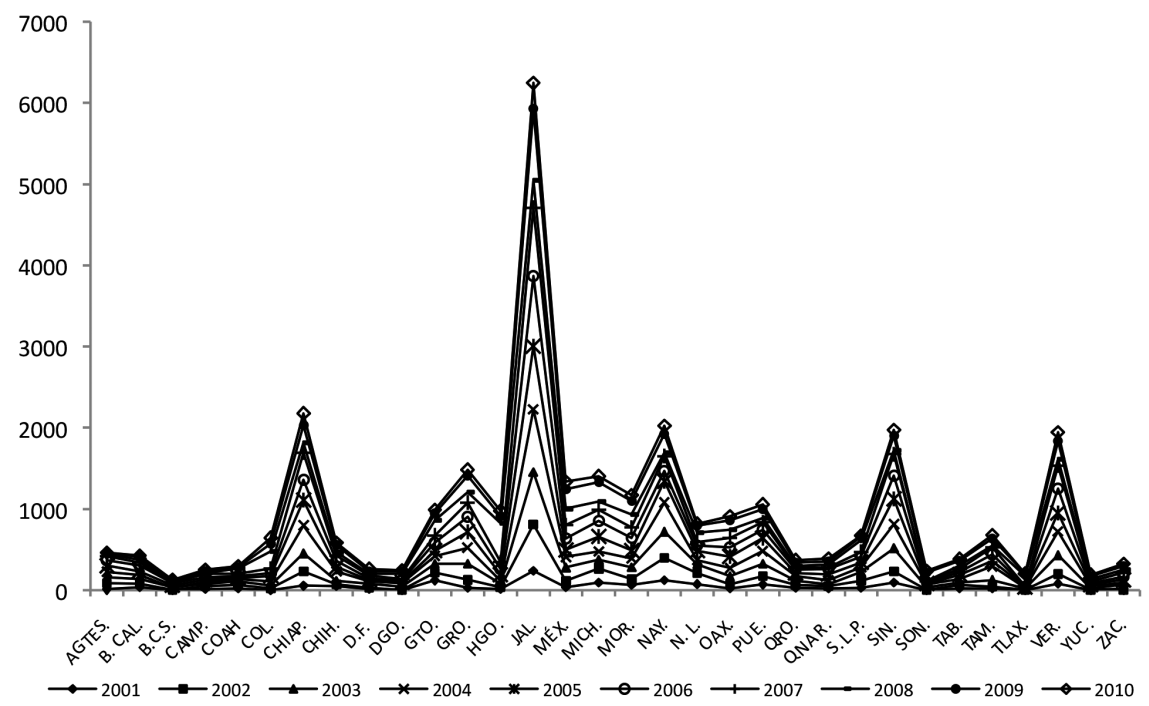

Figura 4. Intoxicaciones por entidad federativa.

En el estado de Jalisco la alta incidencia de registros de intoxicación aguda por plaguicidas, es relacionado al establecimiento de monocultivos, ejemplo de ello es el cultivo del agave azul (Agave tequilana Weber) y caña de azúcar (Saccharum officinarum). Para el primer cultivo, la región de Denominación de Origen reconocida mundialmente, es conformada por cinco estados de la República Mexicana (Jalisco, Michoacán, Tamaulipas, Guanajuato y Nayarit), siendo el estado de Jalisco en donde se puede plantar en la totalidad de sus municipios; con respecto a su manejo, éste de forma tradicional es eliminando cualquier tipo de pasto que emerge en sus parcelas mediante la aplicación de químicos. Para la caña de azúcar, ésta es distribuida principalmente sobre 6 regiones: Norte (Sinaloa), Pacífico (Nayarit, Jalisco, Colima y Michoacán), Centro (Morelos y Puebla), Noroeste (San Luís Potosí y Tamaulipas), Golfo (Veracruz, Tabasco y Oaxaca) y Sur (Chiapas, Campeche y Quintana Roo) (Figura 5).

Basados en el reporte del Comité Nacional para el Desarrollo Sustentable de la Caña de Azúcar (CONADESUCA) con datos de los ingenios 20122013, el estado de Jalisco, es el segundo productor a nivel nacional de azúcar con 535.433,50 t, antecedido 


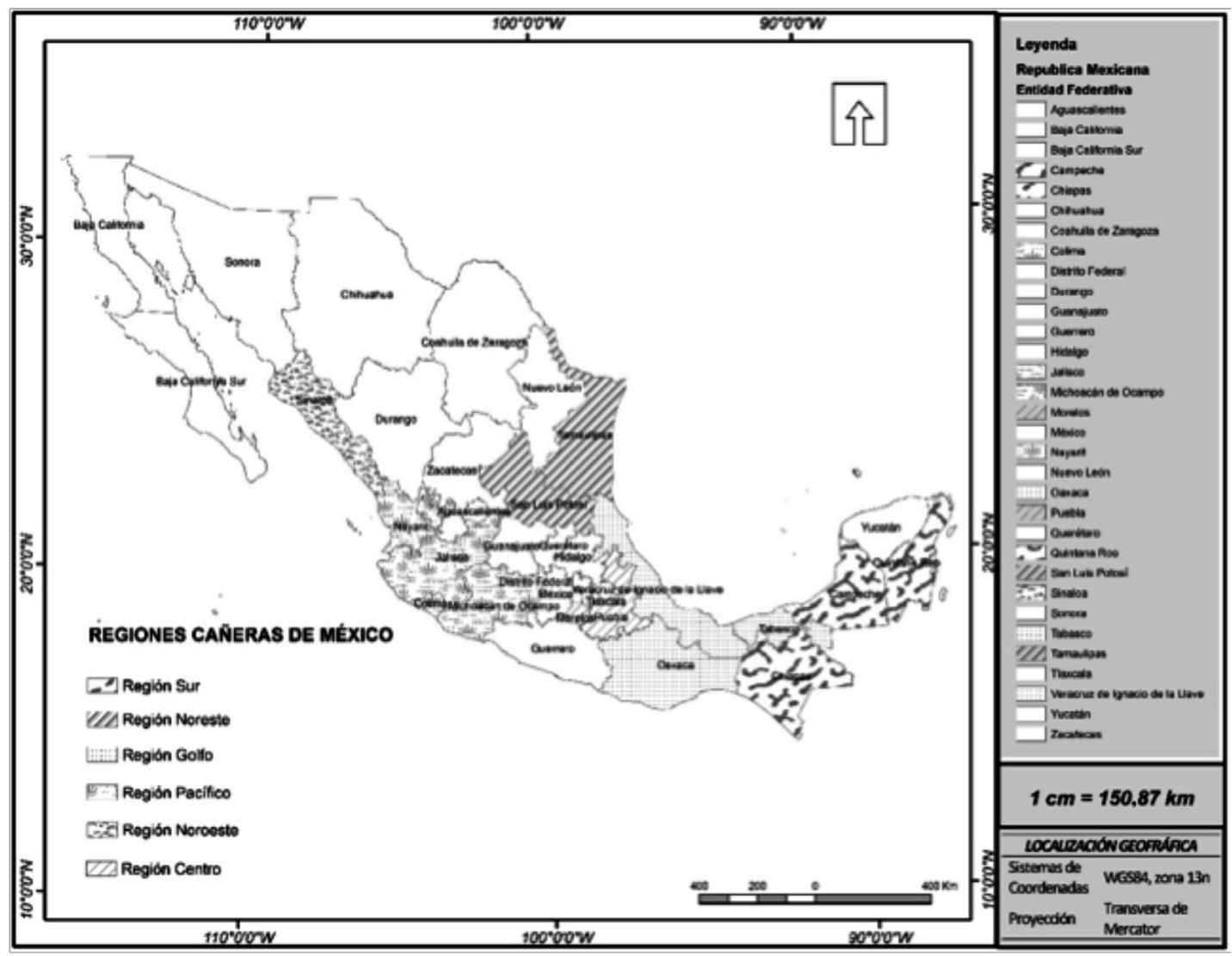

Figura 5. Regiones cañeras de México.

Fuente: Comisión Especial para la Agroindustria Azucarera. Senado de la República.

por Veracruz que produce 1'709.246,62 t; sin embargo, la superficie cosechada de este último es sobre 188.210,33 ha, a diferencia de Jalisco que se cosecha sobre una superficie de 45.730,81 ha; esto hace inferir que la fertilidad de las tierras jaliscienses es muy alta, o que los insumos (agroquímicos) empleados para lograr esta producción tienen mayor frecuencia, situación que puede estar relacionada a la alta incidencia del número de intoxicaciones reportadas para el estado.

\section{Intoxicaciones a nivel regional, Jalisco}

De los reportes de casos de intoxicación por plaguicidas del Sector Salud Regional (IMSS y SSA) de 2004 a 2011 en las regiones Sierra de Amula y Costa Sur del estado de Jalisco, se manifiesta que los municipios con mayor número de casos fueron Autlán de Navarro (459 casos) y Casimiro Castillo (221 casos), ambos identificados por la superficie sembrada con caña de azúcar; el primero de ellos, por contener uno de los valles agrícolas más importantes del estado correspondiente al Distrito de Desarrollo Rural 05, lo que se traduce en el mayor uso de productos químicos, que se refleja en la mayor incidencia de casos de intoxicación (Figura 6).

La respuesta de incidencia y persistencia de intoxicaciones agudas por plaguicidas tiende a incrementarse debido al manejo de monocultivos en estas regiones; ejemplo de ello es el incremento del cultivo caña de azúcar, debido a las demandas de materia prima para las 6 industrias azucareras (ingenios azucareros) establecidas en el Estado de Jalisco. Entre éstas destacan dos por su producción: el Ingenio Melchor Ocampo S.A. de C. V., ubicado en Autlán de Navarro (durante 2013-2014 se registró una superficie total industrializada de 10.120 ha, generando una producción de azúcar de 133.088 t) y el Ingenio José María Morelos S.A. de C.V. en Casimiro Castillo (superficie de corte de 10.084 ha y producción de azúcar de 81.678 t). 


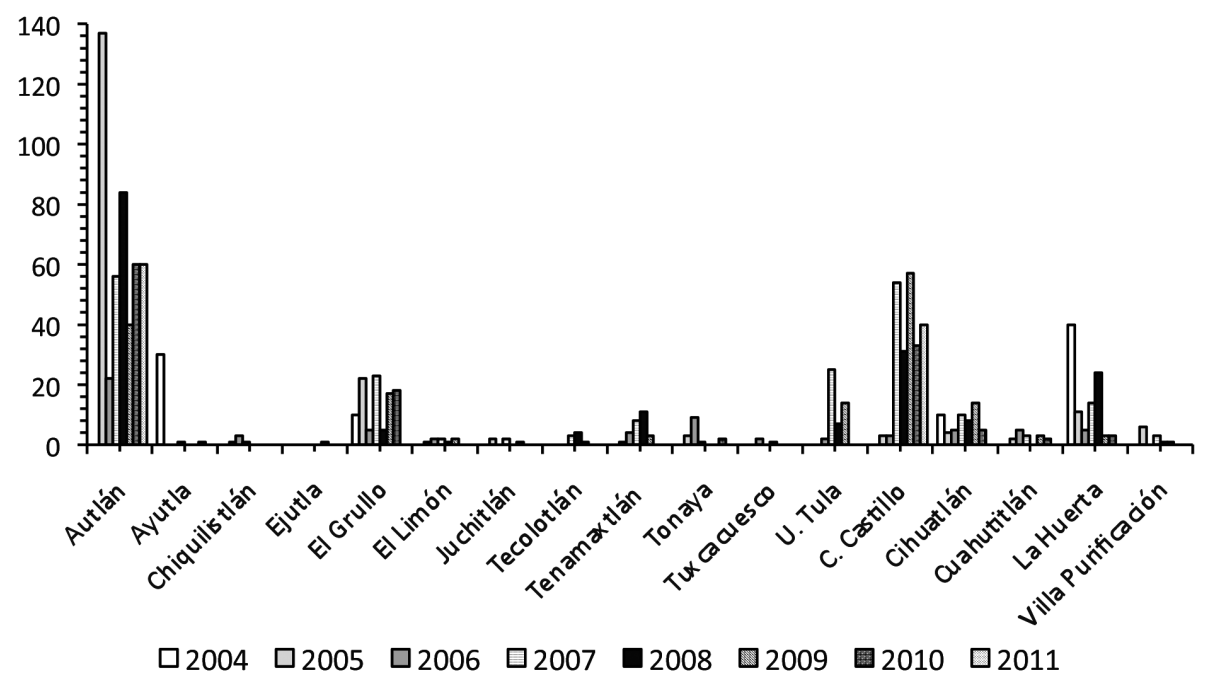

Figura 6. Intoxicaciones agudas por plaguicidas en las Regiones Sierra de Amula y Costa Sur, Estado de Jalisco - México (IMSS y SSA, 2011).

Otro ejemplo bajo el sistema de monocultivo en el municipio de Autlán de Navarro, es el cultivo agave azul (Agave tequilana Weber), establecido de manera extensiva e intensiva durante el periodo 1995-2010, en su momento trajo consigo importantes fuentes de trabajo en diferentes municipios; sin embargo, en su manejo el requerimiento de mayor control de malezas produjo el aumento y la demanda de productos químicos así como la degradación de suelos afectando directa e indirectamente la vegetación nativa (Ej. Huizaches y órganos de pitayo, entre otros), la fauna (Macrofauna y microfauna), pero principalmente la salud de trabajadores en este cultivo que maniobran los productos químicos e interaccionan con el cultivo.

\section{Intoxicaciones a nivel municipal}

Los registros de la Secretaría de Salud en referencia a los casos de intoxicación aguda por plaguicidas para los ejidos del municipio de Autlán de Navarro, fueron de 134 casos en el periodo 2004 a 2011; de 24 ejidos que conforman este municipio, únicamente en cuatro se tienen registros de esta problemática, siendo el ejido de Autlán el de mayor número de casos registrados con incidencia en todos los años de registro (Figura 7). Esta respuesta manifiesta la carencia de información sistematizada a nivel ejido, con respecto a lo reportado para el municipio.

Este comportamiento supone está relacionado al mayor número de cultivos protegidos (invernaderos)

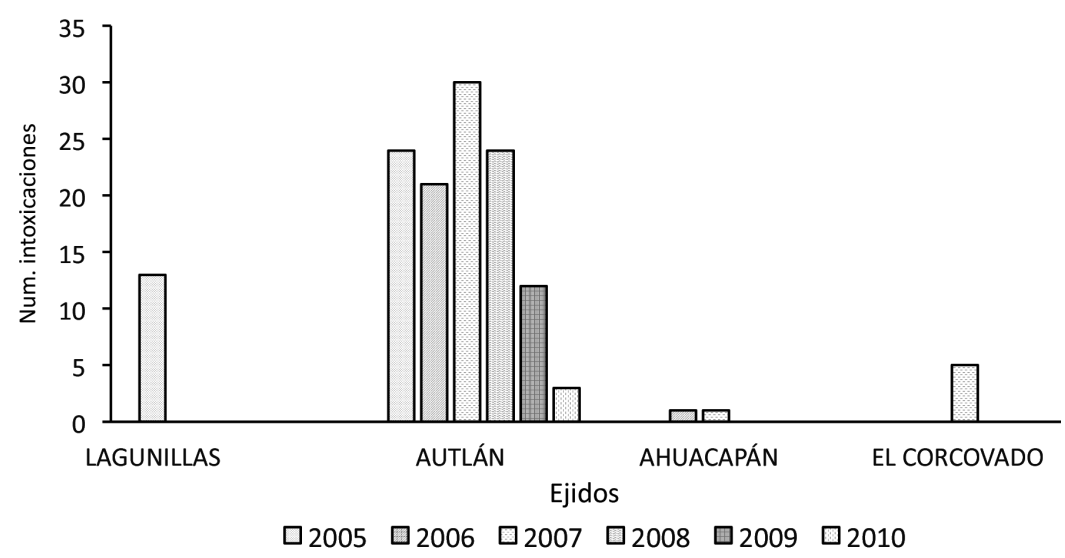

Figura 7. Intoxicaciones agudas por plaguicidas en ejidos del municipio de Autlán de Navarro. 
por empresas ubicados en el ejido de Autlán, estando su producción basada en el uso intensivo de agroquímicos (principalmente en hortalizas). Adicional a ello, se asume la elevada relación de los casos de intoxicaciones con el cultivo de caña de azúcar, por ser el cultivo de mayor extensión en el municipio y el cual requiere un tipo de manejo ya sea a través de mano de obra y aplicación de algunos plaguicidas, en específico herbicidas a lo largo de su desarrollo, productos que provocan menor impacto a diferencia de los insecticidas, pero que de igual forma afectan al ser humano y al ambiente. Basados en Barba-Ho y Becerra (2011) se establece que los herbicidas son usados con mayor frecuencia y de superior grado de toxicidad.

De la información obtenida a través de las encuestas, se identificó a los ejidos de Autlán, El Chante, Ahuacapán y Rincón de Luisa con el mayor número de entrevistas debido al número de ejidatarios registrados para cada ejido; de las encuestas, $22 \%$ de los agricultores manifiestan haber padecido o tuvieron alguna reacción al contacto con agroquímicos al menos en una ocasión; de los 24 ejidos encuestados, en 19 se manifiesta daño a la salud por estos productos, el total de personas afectadas fue de 50 casos, siendo la causa principal el contacto con insecticidas y herbicidas (Figura 8). Los principales insecticidas empleados con mayor frecuencia fueron: Lannate (Metomilo: S-Metil-Nmetilcarbamoil oxi - tioacetamidato), Tamarón (Metamidofos: O, S-Dimetil fosforoamidotioato) y Furadán (Carbofuran: 2, 3-dihidro-2, 2-dimetil7-benzofuranil-metil carbamato) (Tabla 1); con respecto a los herbicidas se identifican 26 nombres comerciales de uso común, los más mencionados son: Faena, Hierbamina, Gramoxone y Caña D (Glifosato). Basados en los informes de la Organización Mundial de la Salud (OMS) estos son considerados en categoría toxicológica muy alta; otro producto empleado en el municipio de categoría toxicológica alta fue Quilt (Tabla 2). En todos los casos de intoxicación por estos productos, fue requerida la atención médica ya que el daño fue grave; a la fecha según estas personas afectadas continúan con secuelas o enfermedades crónicas como cáncer producido por el producto Quilt, o problemas gastrointestinales/digestivos generados por productos como Lannate y Tamarón.

En relación a estos resultados, de la base de datos electrónica e impresa de los registros

Tabla 1. Frecuencia y producto empleado de agroquímico.

\begin{tabular}{lclc}
\hline Producto & Frecuencia & Producto & Frecuencia \\
\hline No recuerda & 18 & Ambush & 1 \\
Lannate & 11 & Cipermetrina & 1 \\
Faena & 4 & Cuit & 1 \\
Furadán & 4 & Esterón & 1 \\
Tamarón & 3 & Karate & 1 \\
Dicteres & 2 & Tordón & 1 \\
Paratión & 1 & Volatón & 1 \\
\hline
\end{tabular}

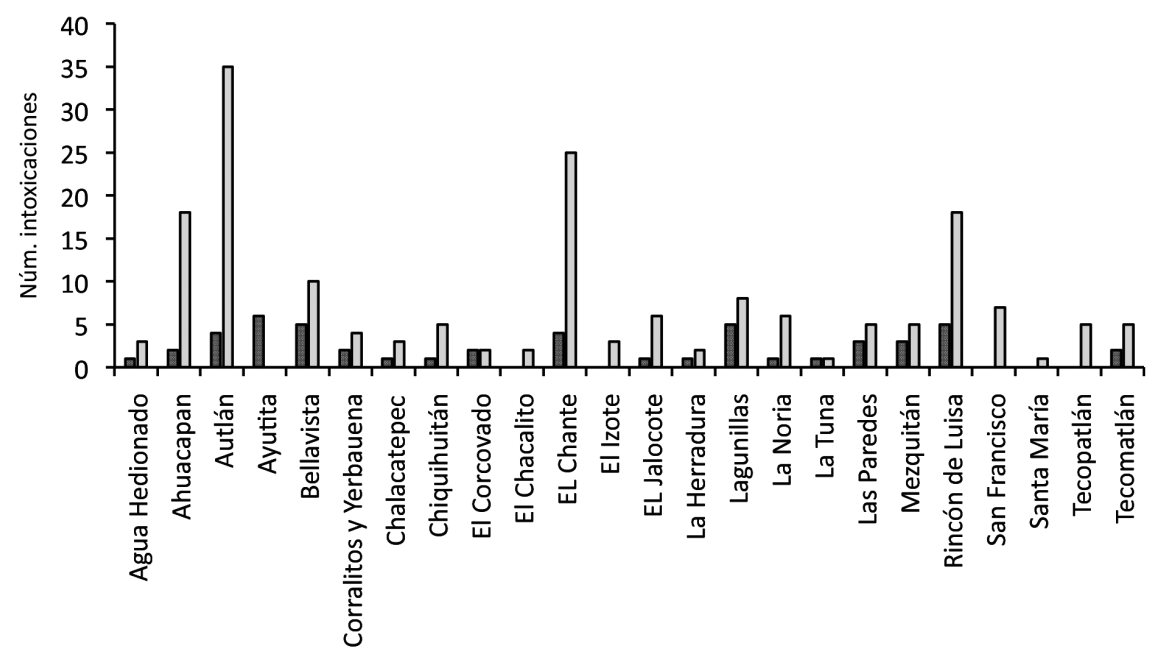

$\square S I \square N O$

Figura 8. Ejidos afectados por el uso de productos químicos. 
Tabla 2. Categoría toxicológica, persistencia y bioacumulación de agroquímicos empleados.

\begin{tabular}{|c|c|c|c|c|c|c|c|c|c|}
\hline \multirow{2}{*}{ 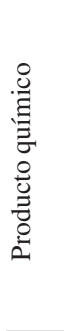 } & \multirow{2}{*}{$\begin{array}{c}\text { Marca } \\
\text { comercial }\end{array}$} & \multirow{2}{*}{ Ingrediente activo } & \multirow{2}{*}{$\begin{array}{l}\dot{\tilde{\Xi}} \\
\dot{\Xi} \\
\dot{\Xi}\end{array}$} & \multirow{2}{*}{ 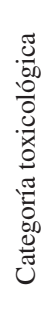 } & \multicolumn{4}{|c|}{ Persistencia (días) } & \multirow{2}{*}{ 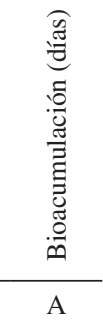 } \\
\hline & & & & & A & $\mathrm{Ai}$ & $\mathrm{Sd}$ & $\mathrm{S}$ & \\
\hline $\mathrm{F}$ & Quilt 200 SE & Propiconazole, Azoxistrobin & $60207-90-1$ & & 60 & 0,7 & 540 & 120 & 130 \\
\hline $\mathrm{H}$ & Dicter & & $131860-33-8$ & & & & & & \\
\hline $\mathrm{H}$ & Esterón 50 D & Ester butílico & $94-80-4$ & III & 38 & 2 & 340 & 75 & 360 \\
\hline $\mathrm{H}$ & Faena 480 & Glifosato, sal isopropilamina & $38641-94-0$ & III & 38 & 0,2 & 340 & 75 & 3,2 \\
\hline $\mathrm{H}$ & Tordón 101 & Picloram & 1918.02 .1 & II & 60 & 19 & 540 & 120 & 3,2 \\
\hline I & Cipermetrina & Cipermetrina & $52315-07-8$ & II & 180 & 0,8 & 1,600 & 360 & 970 \\
\hline I & Furadán 98\% & Carbofuran & $1563-66-2$ & II & 38 & 0,6 & 340 & 75 & 16 \\
\hline I & Karate 50 EC & Lambdacihalotrina & $91465-08-6$ & II & 180 & 0,5 & 1,600 & 360 & 1,100 \\
\hline I & Lannate & Metomil & $16752-77-5$ & I & 15 & 2,4 & 140 & 30 & 3,2 \\
\hline I & Paratión & MetilParation & $298-00-0$ & I & 38 & 0,3 & 340 & 75 & 36 \\
\hline I & Tamarón 600 & Metamidofos & $10265-92-6$ & I & 15 & 0,5 & 140 & 30 & 3,2 \\
\hline I & Volatón DP 3 & Foxim & $14816-18-3$ & III & 38 & 0,2 & 340 & 75 & 370 \\
\hline I & Ambush & Permetrina & $52645-53-1$ & II & & & & & \\
\hline
\end{tabular}

Producto químico: F, fungicida; $\mathbf{H}$, herbicida; $\mathbf{I}$, insecticida.

Persistencia: A, agua; Ai, aire; $\mathbf{S d}$, sedimento; $\mathbf{S}$, suelo.

Num. Cas. Identificación numérica para compuestos químicos que otorga el Servicio de Resúmenes Químicos (Chemical Abstract Service o CAS) de la Sociedad Química Americana.

obtenidos del sector salud (Instituto Mexicano del Seguro Social y Secretaría de Salud y Asistencia Pública) y basado en lo manifiesto por diversos autores (Safe, 1995; Alvarado y Pérez, 1998; Tagliaferro et al., 1999; González y Bernal, 2000; Rivero et al., 2001; Martínez-Valenzuela y GómezArroyo, 2007; Pierre y Betancourt, 2007; Montes et al., 2010; Montoro et al., 2009; Zamora et al., 2009), se establece que el uso de agroquímicos afecta negativamente la salud de los humanos, pero principalmente a los usuarios directos, creándoles daños graves e irremediables.

La comparación entre ejidos así como dentro de ellos, basados en los casos de intoxicación por plaguicidas, no manifiesta haber diferencia entre éstos (Tabla 3). Los resultados reafirman que las intoxicaciones agudas por plaguicidas pueden tener diversas causas: descuidos, mala información y desconocimiento sobre las precauciones de protección en la manipulación de los productos. Sobre todo en mayor medida con los productos altamente tóxicos, lo que repercute directamente en la salud del ser humano, incrementando el riesgo y exposición a terceras personas. En este sentido 93\% de los agricultores menciona que el conocimiento sobre manejo de estos productos ha sido adquirido a través de la experiencia en campo y $3 \%$ menciona que ha recibido capacitación y asesoría. No obstante $65 \%$ de ellos reconocen los riesgos que conlleva el uso de productos químicos, argumentando que se pueden "enyerbar" o intoxicar; riesgo que se incrementa por la falta de equipo especializado, ya que $48 \%$ de los jornales no utilizan equipo para su protección, $41 \%$ afirma que utilizan materiales plásticos (bolsas) para cubrir la espalda como protección, en el mejor de los casos utilizan botas de hule, guantes y un pañuelo o cubre boca para protección de las vías respiratorias (Figura 9); mencionan también que siempre tratan de tener precaución de no comer, fumar o tomar alcohol en horas cercanas o en el momento en que están fumigando, teniendo precaución con la dirección del viento al aplicar los productos y que éstos sean aplicados por las mañanas. Siendo los responsables de llevar a cabo la aplicación del agroquímico los jornaleros (63\%) y los propietarios del cultivo (32\%), porcentaje que se reduce cuando los propietarios por salud o edad (adultos mayores) requiere de jornaleros. 
Tabla 3. Análisis de varianza de las encuestas a los agricultores.

\begin{tabular}{lcccccc}
\hline Origen de las variaciones & $\begin{array}{c}\text { Suma de } \\
\text { cuadrados }\end{array}$ & $\begin{array}{c}\text { Grados de } \\
\text { libertad }\end{array}$ & $\begin{array}{c}\text { Promedio de } \\
\text { los cuadrados }\end{array}$ & F & $\begin{array}{c}\text { Probabilidad } \\
\text { Valor crítico } \\
\text { para F }\end{array}$ \\
\hline Entre grupos & 346,68750 & 1 & 346,68750 & 9,32723 & 0,00375 & 4,05175 \\
Dentro de los grupos & 1709,79167 & 46 & 37,16938 & & & \\
Total & 2056,47917 & 47 & & & & \\
\hline
\end{tabular}

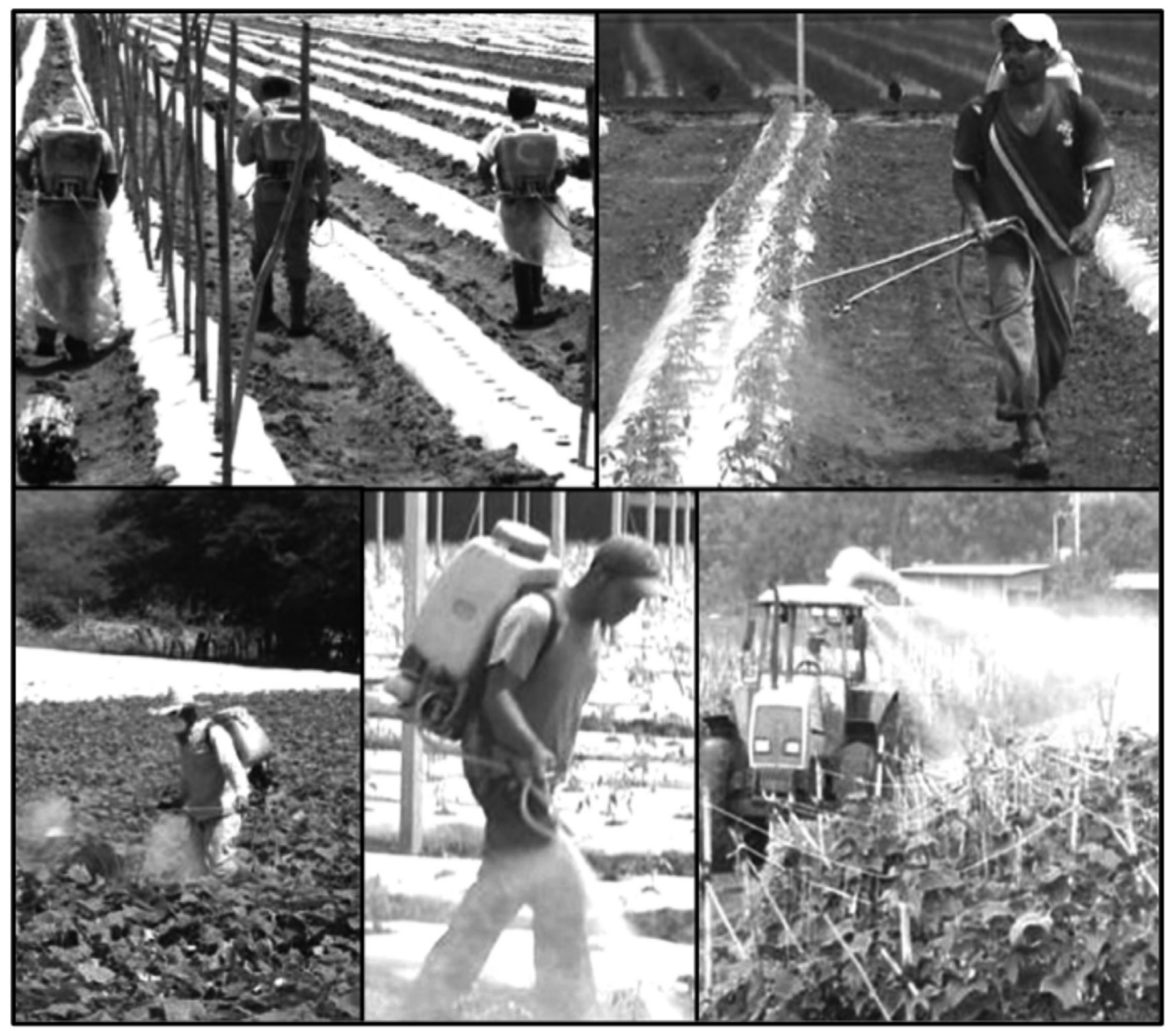

Figura 9. Equipo de protección empleado para la aplicación de agroquímicos.

Finalmente se reconoce que $32 \%$ de las casas comerciales o distribuidores proporcionan información de manejo y uso de los agroquímicos, $37 \%$ no lo hace y $29 \%$ menciona que regularmente brinda esta información. En respuesta a ello, los distribuidores argumentan que los agricultores ya tienen esta información, siendo innecesario brindarla nuevamente a cada compra, ya que la venta de algunos productos es frecuente y con alta demanda de parte del usuario.

\section{Conclusiones}

Se identifica que el riesgo a sufrir intoxicaciones agudas por plaguicidas, está relacionado a la ausencia de: conocimiento técnico, capacitación del manejo y uso de agroquímicos, así como a la información pertinente que el vendedor y el mismo producto proporciona al usuario. Ausencia que ha provocado la reproducción del conocimiento empírico del agricultor, traspasado de padres a hijos y con ello el 
manejo no apropiado del producto. Esta situación ha generado consecuencias irreversibles en la salud del humano y del ambiente. Sin embargo, se considera que previo al riesgo de intoxicación y ante éste, la educación en salud pública permitirá la atención adecuada en su momento para cada caso.

Se considera que el cuidado y protección requerida al manejar los productos agroquímicos, debe ser el más adecuado, las precauciones que sean tomadas al manipularlos reducirán o incrementaran el riesgo según sea el caso de cada usuario. Ante ello, se remarca la necesidad de brindar capacitación sobre su manejo y uso, no exponerse ni exponer a terceros, ya que ello podría traer consecuencias irreversibles en la salud humana de forma directa a través de enfermedades.

En el marco normativo, las implicaciones de distribución, comercialización, manejo y uso de tales productos (restringidos y prohibidos por su grado de toxicidad), muchas ocasiones puede ser desconocido o ignorado por el usuario; conocer y difundir esta legislación, resalta la importancia de contar con estrategias pertinentes que lo promuevan, conformando y manteniendo la concientización sobre el daño que éstos causan al usuario y ante ello las medidas precautorias que mitiguen el riesgo.

Finalmente en el contexto de la sustentabilidad, se enmarca la importancia de investigar, conocer y divulgar las consecuencias que trae consigo la exposición de agroquímicos a nivel local y regional. En referencia a la salud del ser humano, se hace necesario conocer la salud del usuario y ante ello, se recomienda la búsqueda de estrategias y la realización de acciones preventivas de protección, manejo y uso de estos productos, de lo contrario, conocer las consecuencias pero haciendo énfasis en la concientización del usuario.

\section{Literatura Citada}

Aguilar, A.E.P.

2006. Plan Municipal de Desarrollo Rural Sustentable del municipio de Autlán de Navarro. Editorial Municipal de Autlán. Jalisco - México. 68p.

Aguilar, M.J.; Prieto, G.F.; Román, G.A.D.; Monks, S.W.S.; Pulido, F.G.

2007. Niveles de pesticidas organoclorados en Cyprinus carpio (Linnaeus, 1758) del lago de Tecocomulco, Hidalgo, México. En: GALLARDO, J. F. (ed). La contaminación en Iberoamérica. Xenobióticos y metales. Cáceres-España: Sociedad Iberoamericana de Física y Química Ambiental, p. 23-44.

Aranguré, Z.F.J.; Zambrano, C.R.M.; González T.L.; Robledo, M.M.L.

2011. Evaluación de niveles de contaminación por residuos de plaguicidas organoclorados en sedimento y peces, de la presa de la presa de Aguamilpa, Nayarit, México. Waxapa, 1(4): 20-35.

Alvarado, Y.; Pérez, C.

1998. El uso de Biocidas: un problema ambiental. Interciencia, 23(1): 20-25.

Barba-Ho, L.E.; Becerra, D.

2011. Biodegradabilidad y toxicidad de herbicidas utilizados en el cultivo de la caña de azúcar. Ingeniería de Recursos Naturales y del Ambiente, 10: 11-19.

Consejo de Salubridad General.

2011. Prevención primaria, diagnóstico precoz y tratamiento oportuno de la intoxicación aguda por agroquímicos en el primer nivel de atención. Guía Práctica Clínica, Parte 2, 10. México: Secretaría de Salud. 50 p.

Cortés-Genchi, P., A. Villegas-Arrizón, G. Aguilar-Madrid, M.P.

Paz-Román, M. Maruris-Reducindo, C. Juárez-Pérez.

2007. Síntomas ocasionados por plaguicidas en trabajadores agrícolas. Rev. Med. Inst. Mex. Seguro Soc., 46 (2): 145-152.
Ongley, E.D.

1997. Lucha Contra la Contaminación Agrícola de los Recursos Hídricos. Estudio FAO Riego y Drenaje - 55. FAO. Roma, Italia. $154 \mathrm{p}$.

Madeley, J.

2002. Paraquat el controvertido herbicida de Syngenta. Foro Emaús. San Jose, Costa Rica. 53 p.

Martínez-Valenzuela, C.; Gómez-Arroyo, S.

2007. Riesgo genotóxico por exposición a plaguicidas en trabajadores agrícolas. Rev. Int. Contam. Ambient, 23 (4): 185-200.

Montes, B.L.P., Waliszewski S., Hernández-Valero M., Sanín-

Aguirre L., Infanzon-Ruiz R., García, J.A.

2010. Exposición prenatal a los plaguicidas organoclorados y criptorquidia. Ciencia \& Saude Colectiva, 15(1): 1169-1174.

Montoro, Y.; Moreno, R.; Gomero, L.; Reyes, M.

2009. Características de uso de plaguicidas químicos y riesgos para la salud en agricultores de la sierra central del Perú. Revista Peruana de Medicina Experimental y Salud Pública, 26(4): 466-472.

Pierre, F.; Betancourt, P.

2007. Residuos de plaguicidas organoclorados y organofosforados en el cultivo de cebolla en la depresión de Quibor, Venezuela. Bioagro, 19: 69-78.

Rivero, O.; Ponciano P.G.; Oláiz, G.

2001. Daños a la salud humana por plaguicidas, el manual moderno. S.A. de C.V, México D.F.: 488 p.

Safe, S.H.

1995. Environmental and dietary estrogen and human health: Is there a problem? Environ. Health. Perspect, 103: 346-351. 
Tagliaferro, Z.; C. Ludewig, M. Mujica, H. Falcón, E. Ugel, J. Canelón.

1999. Evaluación y vigilancia del uso de plaguicidas sobre la salud de la población del valle de Quibor. Decanato de Medicina. Universidad Centroccidental Lisandro Alvarado. Barquisimeto, Venezuela: $82 \mathrm{p}$.

Torres, D.; Capote, T.

2004. Agroquímicos un problema ambiental global: uso del análisis químico como herramienta para el monitoreo ambiental. Ecosistemas, 13 (3): 2-6.
Waliszewski M.S.; Meza, H.M.; Infanzón, M.R.; Trujillo, M.P.; Morales, G.M.

2003. Niveles de plaguicidas organoclorados persistentes en mujeres con carcinoma mamario en Veracruz. Rev. Int. Contam. Ambient., 19 (2): 59-65.

Zamora, R.F.; Torres, D.; Rodríguez, N.; Zamora, J.F.

2009. Impacto del uso de plaguicidas sobre los niveles de colinesterasa total en sangre en productores agrícolas del asentamiento campesino Santa Teresa, Municipio Miranda del Estado Falcón, Venezuela. Rev. Fac. Agron., 35(2): 56-61. 